\title{
Intelligent corset
}

\author{
P. Aradi, N. Szakály, J. Molnár \& P. Tamás \\ Faculty of Mechanical Engineering, Budapest University of Technology \\ and Economics, Hungary
}

\begin{abstract}
Conservative treatment of spinal disorders, especially scoliosis has been accomplished with plastic or plastic-metal corsets for decades. However, corsets are tailored to the individual's body, the exact distances and action between the patient and the corset are not known. The raw piece is fabricated on the body's exact model, which is made in at least three steps. First, the upper body of a patient set in a predefined posture is plastered, then after drying the plaster is removed. The second step is the preparation of the torso's model that is made of plastic foam covered with plaster. After that the corset is formed according to the desired objectives, namely the orthopaedic technician forms the corset on the torso model and makes the necessary adjustments to achieve the desired spine compensation. The corset is then fitted on the patient and eventually minor corrections are made. New methods have been developed to replace plastering, namely 3D body scanners working with different measurement principles. Traditional corset forming has also been changed to utilize the 3D body model and to create a virtual corset, which can be sent directly to the manufacturing machine. As a last step it might also be necessary to make minor adjustments. As it is said before, there is no measureable data on the interaction of the patient's body and the corset, not even in a static standing position, not to mention during movement, as simple as walking. It is also impossible to determine, whether the patient wears the corset or not, because there are cases when the patients uses the device according to prescription, but there are no improvement in his/her condition or it even gets worse. No one can tell that the changes are because of wearing the tool that is made with the intention to be the best possible or by not using it at all. This paper is about the development of an intelligent battery powered measurement and data collection system for corsets to determine forces between patient and corset at selected points, to detect the corset's position, to measure temperature, and to save the collected data to internal memory. The
\end{abstract}


equipment can be connected to PCs, in order to download and analyze collected data, such as time of wearing, force changes in a timeframe, extreme forces in different life situations. These data help make the corset more appropriate for the intended correction. Further investigations are made with magnetically attached sensors in dynamic motion tests, such as walking, to determine the movement of selected anatomical body points that are not detectable with the motion capture system through the plastic corset.

This paper's main contribution is to show the novel data acquisition and processing system to help making corsets used in the treatment of spinal disorders, especially scoliosis, more effective.

Keywords: biomechatronics, spinal disorders, intelligent measurement, magnetic sensors, intelligent corset.

\section{Introduction}

Part of conservative treatment methods for certain types of spinal disorders has been the use of plastic or plastic-metal corsets. Scoliosis, when the spine is sideways curved is one such problem. Nowadays scoliosis is quite widespread, especially in young girls.

Early detection of scoliosis and corrective measures are very important for effective treatment. As each and every scoliosis patient is different, not just in anatomical, physiological, pathological aspects, but in lifestyle, determination and strength of will, the need to make the correction device, i.e. the corset individually suited is extremely important. Corsets have always been tailored to fit the wearer's body and to provide the necessary correction to achieve the desired posture. However, there was no method to check, whether the corset is worn as prescribed, not to mention physical parameters, e.g. the forces between the corset and its wearer, temperature. The movement of selected anatomical points during dynamic motion is also problematic, when the motion caption system is not able to "see" through the corset.

\section{Physical parameters and their measurement}

\subsection{Temperature}

A simple thermometer IC was chosen to measure the wearer's temperature. NXP's LM75A is a "digital temperature sensor and thermal watchdog" according to the data sheet. $\mathrm{I}^{2} \mathrm{C}$ bus communication is supported.

Together with motion detection normal human body temperature is a good indicator of the prescribed use of the corset.

\subsection{Motion}

Movement, that is whether the corset is on the patient or it is just lying somewhere in a corner can be detected with an accelerometer, or gyroscope. In 
version one and two of the corset electronics the low-cost 3-axis digital output acceleration sensor, Freescale’s MMA7455 was chosen.

\subsection{Force}

Certain connection points between the corset and the human body have great importance to achieve the desired corrective effect. That is why it is important to measure force between the patient and corset. Force sensors for this task have to be lightweight and thin enough not to modify the connecting parts made of plastic and have electrically connectable material. There is no need for very precise force measurement, because the prototype corset electronics needs to detect only one of four cases, "not touching", "too small force", "normal force”, and "too much force". Five or more digits of precision in force measurement is not necessary at the moment.

Interlink Electronics' "Force Sensing Resistor" (FSR) is made from a thin polymer film and conductive material [4]. Resistance changes when force is applied on their area, so not just force, but pressure is detectable, too. The $0.55 \mathrm{~mm}$ thickness of plastic FSR sensors is fixed to the inside of the corset with adhesive material. However, because of the mechanical properties of the sensor's polymer material, and the electrical resistance are not linear, further thorough investigations had to be conducted to prepare a suitable calibration protocol [5].

\section{Corset-mounted data acquisition and storage system}

\subsection{Electronics panel}

Sensors had to be integrated into a data acquisition and storage system that can be mounted to the corset without disturbing the wearer. The custom made electronic panel has an onboard microcontroller (PIC 18F series) to coordinate the sensors by specifying their sampling rate, collect and store their data and when needed transmit them for analysis to a PC.

After making a breadboard model of the electronics, two PCB (printed circuit board) panels were designed. The first version (Figures 1 and 2) was too big, there were two DC-DC converters and it was hot-swappable between battery power and USB power. The analogue part worked from $+5 \mathrm{~V}$ stabilized, the digital part worked from $+3.3 \mathrm{~V}$ converted supply.

A sweat sensor has been planned into the corset, and its place in the panel is reserved, but at the moment it is not implemented. Force sensors, thermometer and accelerometer are installed beside the processor, memory, USB connection and power supply.

The device is battery operated, so the measurement time is limited by the analogue part's power supply. PCB (Printed Circuit Board) is mounted to the corset M3 screws. 


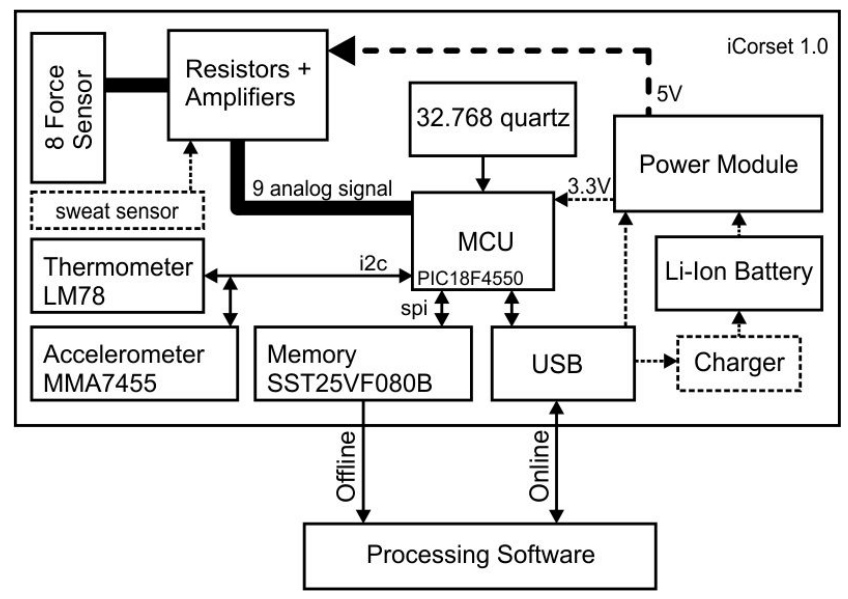

Figure 1: $\quad$ First PCB schematics.

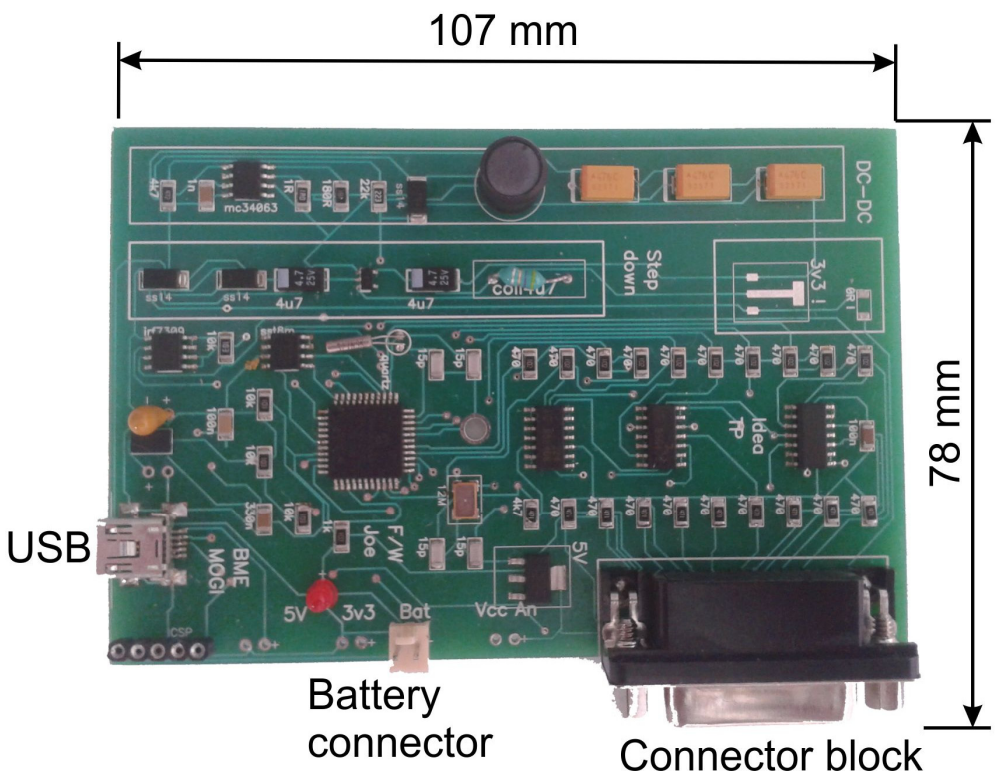

Figure 2: $\quad$ First PCB version.

The second smaller panel (Figures 3 and 4) is used for extensive testing now. It works with a single $+3.3 \mathrm{~V}$ power supply, stabilized by SMD (Surface Mount Device) linear stabilizer, but power is not hot-swappable, as it was in the first version. In this version sensors are directly connected to CPU. The smaller PCB is $40 \times 36 \mathrm{~mm}$ and it is easier to mount on the corset surface, where sometimes there is hardly a big enough flat surface for the previous PCB version.

The second PCB version is mounted to the corset similarly to the first version's M3 screws. 


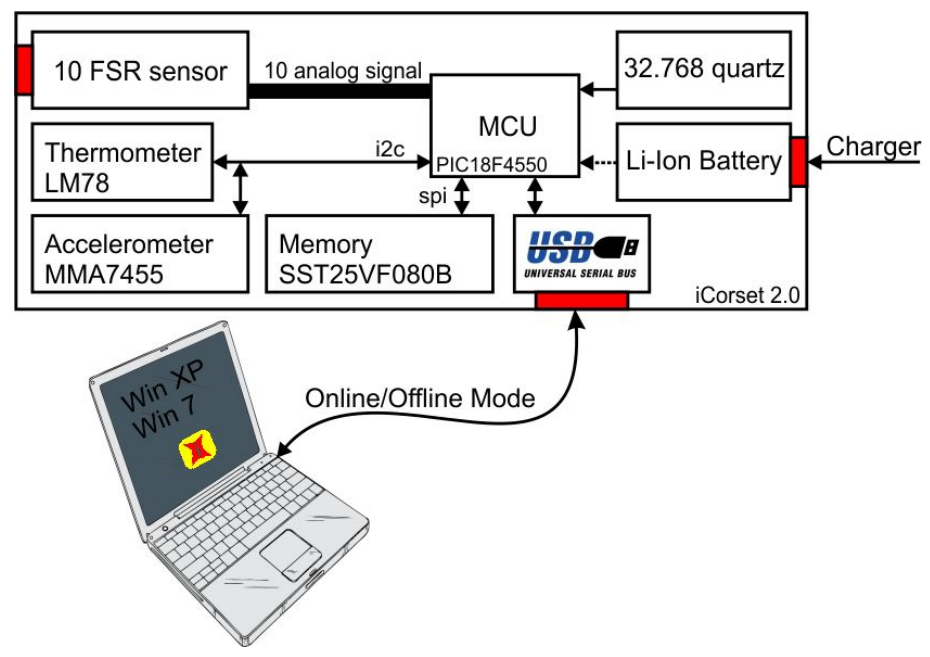

Figure 3: $\quad$ Second PCB schematics.

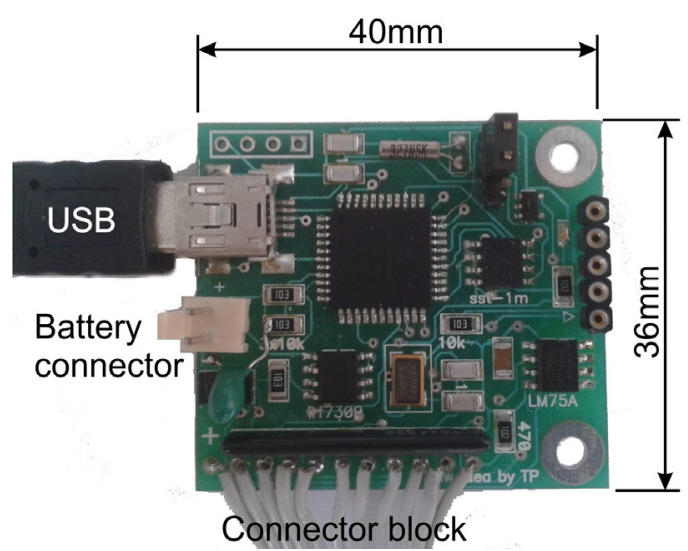

Figure 4: $\quad$ Second PCB with connectors.

\subsection{Sensor calibration}

Chosen force sensors' measurement range is for masses between 10 grams and 10 kilograms. There are no previous measurement data from forces between the body and the corset, however maximal forces are restricted by the tolerance of the biological system. In interconnecting corset-body systems forces are expected to be well below the maximal measurement limit.

A calibration process had to be developed with a standard series of weights (Figure 5), to adjust later data processing. 


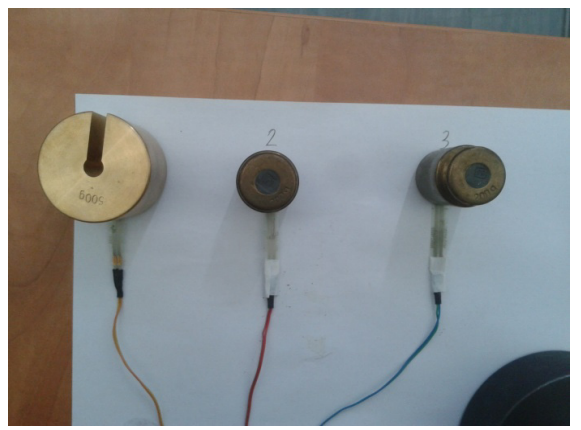

Figure 5: $\quad$ Force sensor calibration.

It is still not thoroughly worked out, how the measured values depend on time and how can their up-down hysteresis be given by a mathematical formula [6, 7]. Sensor properties are interpolated with polynomial and logarithmic functions as shown in Figure 6.

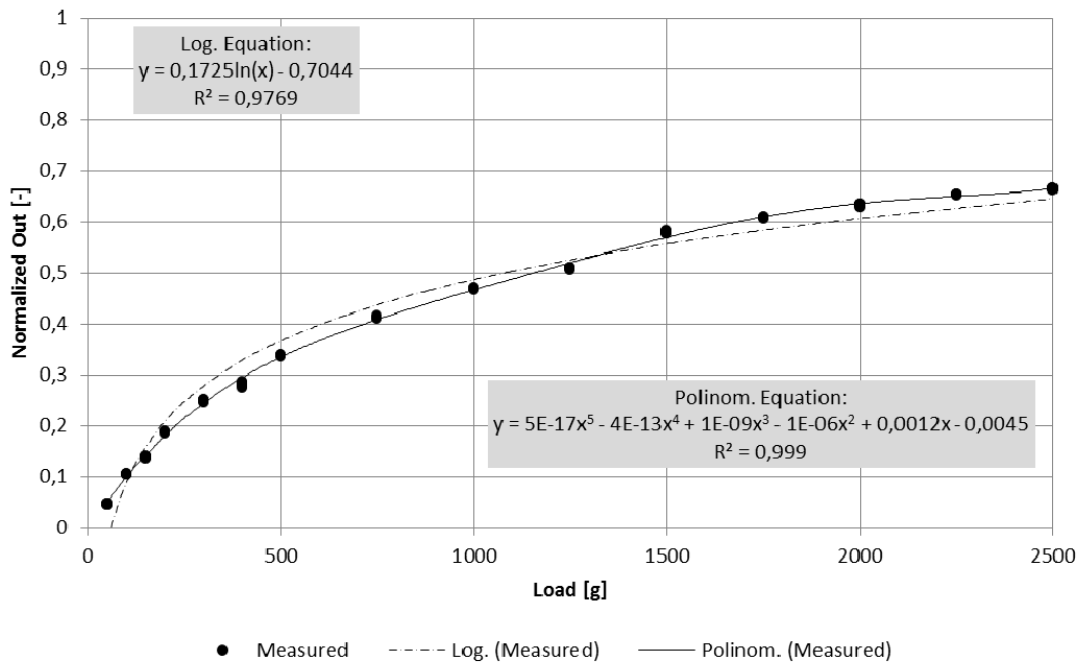

Figure 6: $\quad$ Force sensor calibration diagram with polynomial interpolation.

Experiments are carried out aiming the standardization of the calibration process at the time of this writing.

\subsection{PC connectivity}

Data is transmitted to the PC with standard USB connection, because the corsetmounted panel does not contain any display elements (mostly because of energy 
conservations aspects). That is why the external processing and visualization software is so important.

\section{Data processing and visualization on a PC}

A Windows-based data processing and visualization application was developed. On-line measurement data is displayed real-time, off-line (collected) measurement data is downloaded, than processed.

This application is also used to calibrate sensors, as shown in Figure 7.

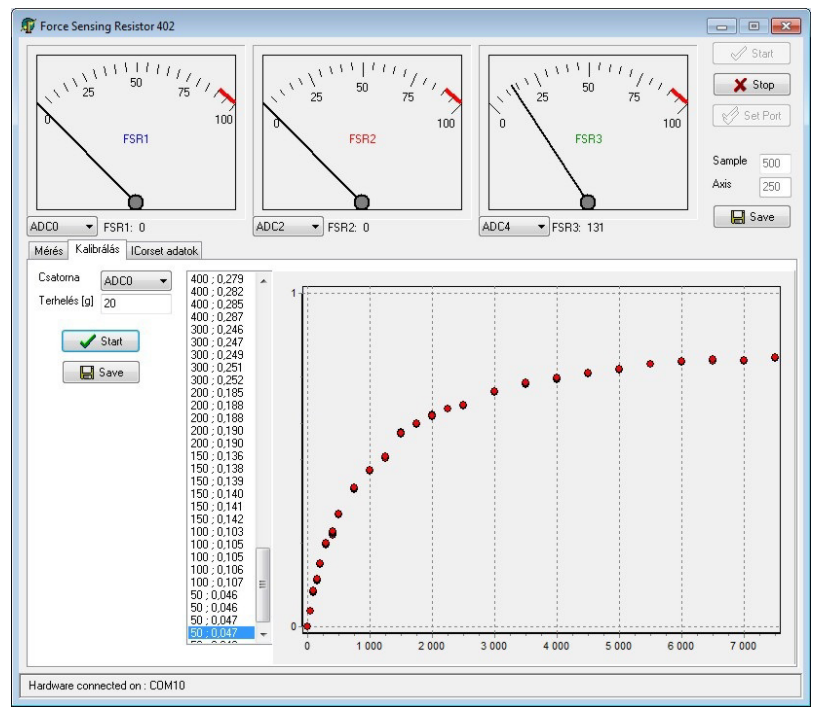

Figure 7: $\quad$ Sensor calibration in the PC application.

\section{Data acquisition is everyday corset use}

The corset equipped with sensors and the onboard electronics panel was and is still extensively tested is as many real world situations as possible. An example is shown below with the first PCB version (Figure 8). Data collected in one week periods are collected, processed and compared now. Corset was used during daytime, in work, free-time activities, but not during night's rest. Patient used her corset both when walking and by travelling by different types of vehicles. Activities were also logged in a conventional diary, so that the corset usage could be compared to the various measured data. The PC application was used to register the corset usage as shown by the sensors. Electronic and conventional logs were compared to help developing both the hardware (sensors and the data acquisition system) and the PC software. 


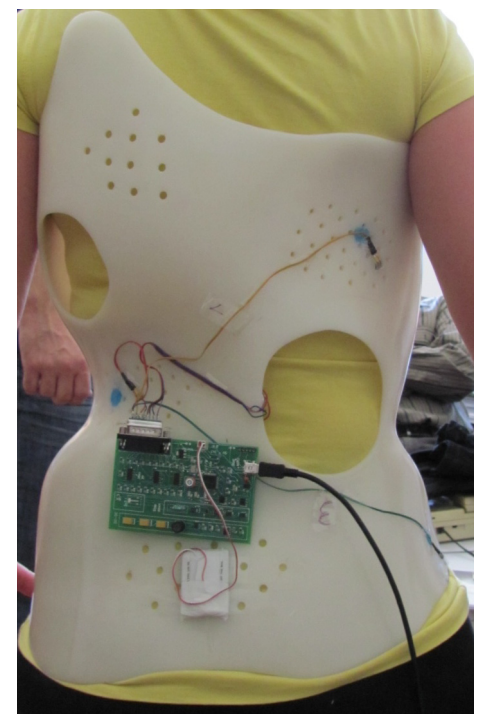

Figure 8: $\quad$ First PCB and sensors mounted to corset and connected to PC via USB.

\section{Dynamic motion tests}

Dynamic motion test were also carried out with motion capture systems. People tested with these systems wear special markers attached to specified anatomic points of their body, as shown in Figure 9, so that the motion capture system can follow their movement during walking. The motion capture system

When the effects of a rigid plastic corset on walking are to be measured the attachment of markers can be a problem, if the corset is not transparent for the motion capture system's cameras. If the marker is put on the outside of the corset at the specified anatomic point, it does not show the exact motion of the body part to be followed.

That is why a magnetic marker attachment method was developed, so the anatomic points on the body surface are so to say extrapolated to the external surface of the corset with the use of magnets (Figure 10), so the six infrared cameras of the motion capture system have no problem in following the markers. A recent BSc thesis [8] dealt with the types and dimension of magnets to be used for such measurements, as well as pilot measurements. 


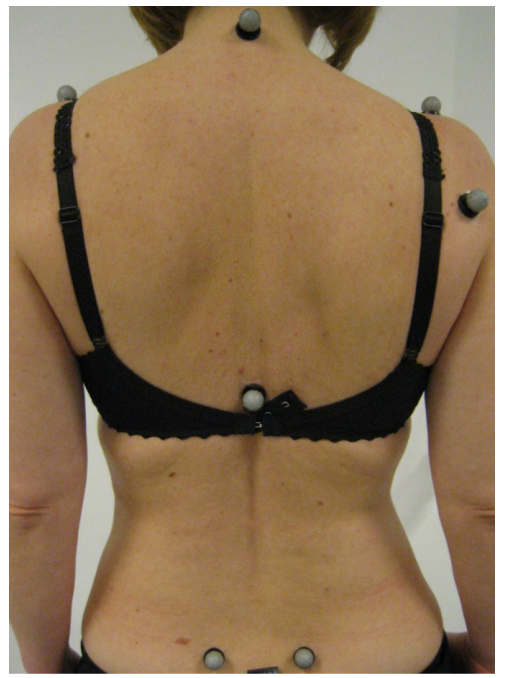

Figure 9: Motion capture system markers attached to the skin.

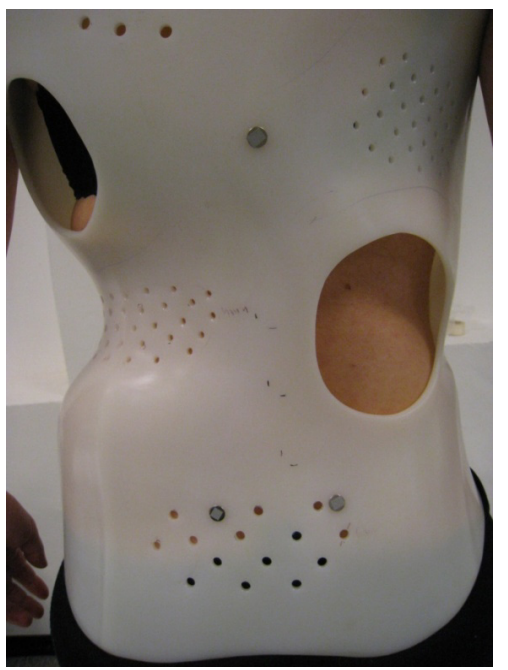

Figure 10: Markers magnetically attached to anatomical points on the skin under the plastic corset.

The result of a test measurements with the corset and the magnetically attached body point markers are shown below. The wavy quasi parallel lines in Figure 11 reflect the movement of selected anatomical points hidden by the nontransparent corset. The lines were recorded during walking and adequately represent the movement of the chosen body points. 


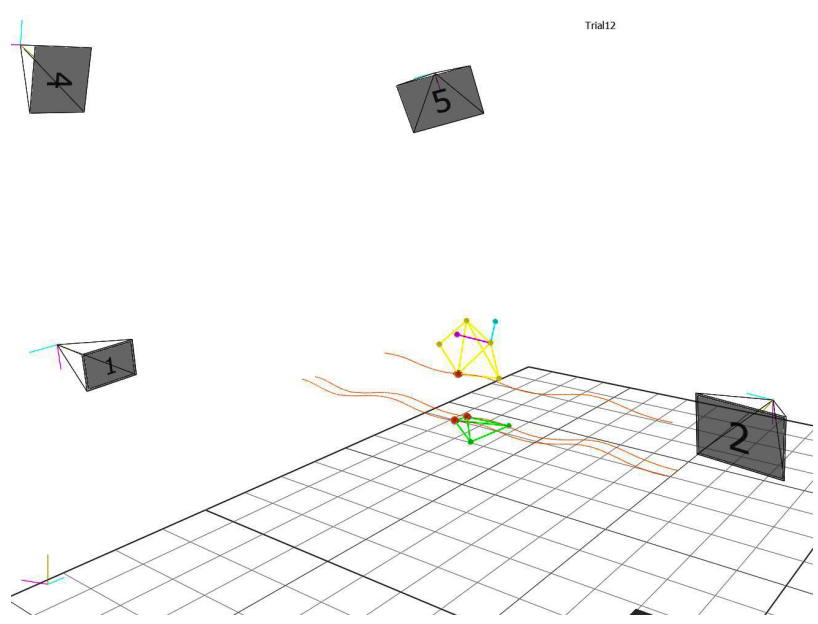

Figure 11: Test with the motion capture system.

\section{Conclusion and further work}

The above mentioned corset mounted sensor system and the processing software, as well as the magnetically attached motion capture markers have further to be tested. An increasing number of corsets are equipped with the sensor system to gather large amount of data. After processing the data both hardware and software will most probably be refined.

Long range results are expected in the area of corset customization, testing the prescribed usage and hereby the effects of corsets.

\section{Acknowledgement}

The authors would like to thank National Development Agency (NDA) of Hungarian Government for its support since this study has been carried out commonly as part of project GERINCO2 TECH_08-A1/2-2008-0121.

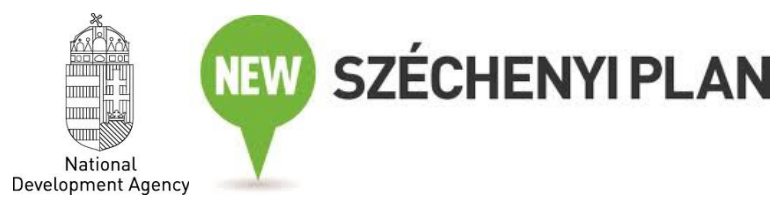

\section{References}

[1] Theodoros B. Grivas (Ed.): Recent Advances in Scoliosis. ISBN 978-95351-0595-4, http://www.intechopen.com/books/recent-advances-in-scoliosis.

[2] Wick, Maureen Jane; Konze, Julie; Alexander, Kelly; Sweeney, Chris: Infantile and Juvenile Scoliosis: The Crooked Path to Diagnosis and Treatment. AORN 90/3 pp. 347-380. 
[3] P.A.F. Martinns, L. Kwiatkowski, V. Franzen, A.E. Tekkaya, M. Kleiner: Single Point Incremental Forming of Polymers, CIRP Annals Manufacturing Technology. CIRP 3962009 Articles in Press.

[4] Interlink Electronics. FSR - Force Sensing Resistor Integration Guide and Evaluation Parts Catalog. Version 1.0, Revision D.

[5] Avrum Hollinger, Marcelo M. Wanderley: Evaluation of Commercial ForceSensing Resistors, NIME06, Paris, France.

[6] J.A Flórez, A. Velásquez: Calibration of Force sensing resistors (fsr) for static and dynamic applications. ANDESCON, 2010 IEEE.

[7] Alexandru Barnea, Cezar Oprisan, Dumitru Olaru: Force Sensitive Resistors Calibration For The Usage In Gripping Devices, Diagnosis And Prediction In Mechanical Engineering Systems, DIPRE 12.

[8] Horváth, Gábor István: Motion capture through non-transparent material, BSc thesis in Mechatronics (major in Biomechatronics), Budapest University of Technology and Economics, Department of Mechatronics, Optics and Engineering Informatics, 2012. 